\title{
Comparative study of screened interlayer interactions in the Coulomb drag effect in bilayer electron systems
}

\author{
R. Asgari, ${ }^{1}$ B. Tanatar, ${ }^{2}$ and B. Davoudi ${ }^{3}$ \\ ${ }^{1}$ School of Physics, Institute for Studies in Theoretical Physics and Mathematics, P.O. Box 19395-5531 Tehran, Iran \\ ${ }^{2}$ Department of Physics, Bilkent University, Bilkent, 06800 Ankara, Turkey \\ ${ }^{3}$ Départment de Physique and Centre de Recherche en Physique du Solide, Université de Sherbrooke, Sherbrooke, \\ Québec, Canada J1K 2R1
}

(Received 21 May 2007; revised manuscript received 6 November 2007; published 3 March 2008)

\begin{abstract}
Coulomb drag experiments in which the interlayer resistivity is measured are important as they provide information on the Coulomb interactions in bilayer systems. When the layer densities are low correlation effects become significant to account for the quantitative description of experimental results. We investigate systematically various models of effective interlayer interactions in a bilayer system and compare our results with recent experiments. In the low density regime, the correlation effects are included via the intra- and interlayer local-field corrections. We employ several theoretical approaches to construct static local-field corrections. Our comparative study demonstrates the importance of including the correlation effects accurately in the calculation of drag resistivity. Recent experiments performed at low layer densities are adequately described by effective interlayer interactions incorporating static correlations.
\end{abstract}

DOI: 10.1103/PhysRevB.77.115301

PACS number(s): 73.21.Ac, 73.40.Kp

\section{INTRODUCTION}

In the past decade transport properties of dilute twodimensional (2D) electron and hole systems have amassed a great interest. Much of the excitement and controversy is centered around the temperature dependence of resistivity which appears to exhibit metallic behavior at high densities and insulating behavior at low densities. ${ }^{1}$ In bilayer systems in which the barrier separating the coupled quantum wells is large enough so that tunneling effects are negligible, the interlayer resistivity has been measured for more than a decade. ${ }^{2}$ In this so-called drag effect the momentum transfer between the layers is measured. ${ }^{3}$ In contrast to the single layer resistivity which shows a nontrivial interplay between interaction and disorder effects near the metal-insulator transition, ${ }^{4}$ the interlayer resistivity is largely determined by the long range Coulomb scattering (as long as the single layer densities are away from metal-insulator transition region). Therefore Coulomb drag experiments provide valuable information on the intra- and interlayer electron-electron interactions especially when the layer densities are lowered.

Over the years there has been a number of Coulomb drag experiments at zero magnetic field using different samples and probing different parameter regimes. The main parameters entering a drag experiment setup are the layer density $n$ which may be related to the dimensionless coupling strength $r_{s}$ (for the definition of $r_{s}$, see Sec. II), the separation distance between the layers $d$, and the Fermi temperature $T_{F}$. Hill et $a l .{ }^{5}$ measured drag resistivity $\rho_{D}$ in an electron bilayer system at densities corresponding to $1.13 \lesssim r_{s} \lesssim 1.57$ and high temperatures $T \sim T_{F}$. The observed peak in $\rho_{D}$ around $T$ $\approx T_{F} / 2$ was attributed to the contribution of plasmons. In fact, the experimental results were regarded as an indirect evidence for the existence of acoustic and optical plasmons in a bilayer system. ${ }^{6}$ Similar experiments were also performed by Noh et al..$^{7}$ confirming plasmon effects on the drag resistivity and revealing the importance of possible dy- namic correlations even though the layer densities were such that $r_{s} \approx 1.48$ where the strong coupling effects are not expected. More recent experiments by Kellogg et al. ${ }^{8}$ used samples with layer densities reaching $r_{s} \approx 4.3$ and $k_{F} d \sim 1$ where $d$ is the center-to-center well separation. In contrast to the above experiments, Pillarisetty et al. ${ }^{9}$ measured frictional drag between two dilute 2D hole layers in which the $r_{s}$ values were in the range $19 \leqslant r_{s} \leqslant 39$.

On the theoretical side, the drag resistivity has first been formulated within the random-phase approximation (RPA) for the layer density-response functions and interlayer effective interaction. ${ }^{10,11}$ Here and most subsequent works treat the interlayer effective interaction as given by the bare interlayer Coulomb interaction screened by the bilayer system dielectric function. Importance of dynamical correlations is noticed even at the RPA level since the difference between the static and dynamic screening function brings quantitative changes to the drag resistivity. ${ }^{10}$ At larger $r_{s}$ values when the correlation effects become significant one should go beyond the RPA. One way to do this in a physically motivated way is through the local-field corrections to the RPA form of the screening function. The simplest form of the local-field corrections is the Hubbard approximation which was used by Hill et al. ${ }^{5}$ to analyze their data. Much widely used localfield corrections are calculated within the self-consistent field approximation scheme of Singwi et al. ${ }^{12}$ (STLS). They have been incorporated in the evaluation of the drag resistivity by Świerkowski et al. ${ }^{13}$ In connection with the experiments of Kellogg et al., ${ }^{8}$ Yurtsever et al. ${ }^{14}$ pointed out that STLS local-field corrections yield a poor representation and suggested the use of a different effective interaction originally developed by Kukkonen and Overhauser ${ }^{15}$ and Vignale and Singwi. ${ }^{16}$ Recently, Badalyan et al. ${ }^{17}$ employed frequency dependent local-field corrections in the long-wavelength limit $(q \rightarrow 0)$ obtained from dynamical exchange-correlation kernel in the context of density functional theory.

In this work we investigate systematically the effect of the form of screened interlayer interaction on the temperature 
dependence of drag resistivity. We calculate the drag resistivity employing several models for the interlayer interaction and compare their behavior with the experimental results of Kellogg et al. ${ }^{8}$ which provide a useful test at low density. As input to various theoretical models of interlayer interaction we consider several constructs of local-field corrections. Our calculations reveal the importance of the choice of interlayer interaction model and the significant role played by the localfield corrections.

The rest of this paper is organized as follows. In Sec. II, we introduce the models for interlayer interaction that enters the drag resistivity. We then outline the calculation of localfield corrections in various approaches. Section III contains our numerical calculations of drag resistivity and comparison of models with experimental data. We conclude in Sec. IV with a brief summary.

\section{THEORETICAL APPROACH}

We consider a double-quantum-well structure with $d$ as the center-to-center well separation such that there is no tunneling between them and $L$ as the width of the quantum wells. Each layer is characterized by the dimensionless coupling constant $r_{s} a_{B}^{*}=1 / \sqrt{\pi n}$ where $n$ is the areal density, $a_{B}^{*}$ $=\hbar^{2} \epsilon /\left(m^{*} e^{2}\right)$ is the effective Bohr radius, and $\epsilon$ and $m^{*}$ being the background dielectric constant and electron band effective mass. Each layer has only one type of charge carrier, i.e., electrons, although our theoretical formulation could be applicable to hole-hole and electron-hole layers with suitable changes. In the case of electron-hole bilayers the prospect of formation of an excitonic state ${ }^{18}$ and its detection through drag experiments ${ }^{19}$ requires a new formulation of the effective interlayer interaction which we do not address here. However, correlations in electron-hole bilayers and their effects on drag resistivity can be studied using the improved interlayer models we shall describe below. The motion of the carriers is free along the $x y$ plane and under the action of a double-well potential profile in the $z$ direction only the lowest subband in each quantum well is occupied. For this aim, temperature should be less than the difference between excited energy level and the ground state energy in quantum well. This yields $T<3\left(r_{s} a_{B}^{*} / L\right)^{2} T_{F} / 16$. Furthermore, the bilayer system is assumed to be embedded in a uniform neutralizing positive background charge. The unscreened Coulomb interaction potential, in Fourier space, between the electrons in $k$ th and $l$ th layers is given by $v_{k l}(q)=v_{q} F_{k l}(q L)$. Here, $v_{q}=2 \pi e^{2} /(\epsilon q)$ and $F_{k l}$ are infinite quantum-well form factors taking the finite width effects into account which are given by ${ }^{10}$

$$
\begin{gathered}
F_{k k}(x)=\frac{3 x+8 \pi^{2} / x}{x^{2}+4 \pi^{2}}-\frac{32 \pi^{4}[1-\exp (-x)]}{x^{2}\left(x^{2}+4 \pi^{2}\right)^{2}}, \\
F_{k l}(x)=\frac{64 \pi^{4} \sinh ^{2}(x / 2)}{x^{2}\left(x^{2}+4 \pi^{2}\right)^{2}} \exp (-q d) .
\end{gathered}
$$

We note that most theoretical calculations ${ }^{6,10,13}$ adopt the infinite quantum-well model to account for the width effects, whereas a better way would be to calculate the Coulomb matrix elements using envelope functions $\phi_{n}(z)$ determined self-consistently from the Poisson and Schrödinger equations. ${ }^{20}$

The drag resistivity (or as it is also called transresistivity) $\rho_{D}$ of an electron system at temperature $T$ has been obtained in a variety of theoretical models. These include diagrammatic perturbation theory, ${ }^{10,21}$ the Boltzmann equation, ${ }^{22}$ and the memory function formalism. ${ }^{11,13}$ In a drag experiment one applies an electric field $E_{1}$ to layer 1 (drive layer) creating a current to flow with current density $J_{1}$. This sets up an electric field $E_{2}$ in layer 2 (drag layer) where no current is allowed to flow. The drag resistivity is defined as $\rho_{D}$ $=E_{2} / J_{1}$ and the microscopic calculations relate this quantity to the rate of change of momentum between the layers, as electron-electron interlayer interactions transfer momentum from the drive layer with carrier density $n_{1}$ to the drag layer with density $n_{2}$.

Theoretical considerations lead to the same expression for $\rho_{D}$ in terms of the effective interlayer interaction and the density-response function of the single layers. When the effective interlayer interaction treated perturbatively, $\rho_{D}$ is given as

$$
\begin{aligned}
\rho_{D}= & -\frac{\hbar^{2}}{8 \pi^{2} e^{2} n_{1} n_{2} k_{B} T} \int_{0}^{\infty} q^{3} d q \\
& \times \int_{0}^{\infty} d \omega \frac{\left|W_{12}(q, \omega)\right|^{2} \operatorname{Im} \chi_{1}^{0}(q, \omega, T) \operatorname{Im} \chi_{2}^{0}(q, \omega, T)}{\sinh ^{2}\left(\hbar \omega / 2 k_{B} T\right)},
\end{aligned}
$$

where $\chi_{i}^{0}(q, \omega),(i=1$ or 2$)$ is the noninteracting linear response corresponding to the drive and drag layer which shows the charge density fluctuations in a given layer at finite temperature and $W_{12}(q, \omega)$ is the effective interlayer interaction.

An important ingredient which is needed to calculate $\rho_{D}$ is the electron-electron interlayer interaction, $W_{12}(q, \omega)$. The effective electron-electron interaction for a two-component system given by a $2 \times 2$ matrix and in RPA is given by

$$
\hat{W}_{R P A}(q, \omega)=\hat{v}(q)+\hat{v}(q) \hat{\chi}(q, \omega) \hat{v}(q),
$$

where $\hat{\chi}(q, \omega)$ is defined in terms of the noninteracting charge-charge response function and Coulomb interactions.

To take into account the effect of correlations more clearly, which are more important in the strongly correlated regime where $r_{s}$ becomes large, we need more sophisticated approaches. For this purpose, we introduce here other approximation scheme for $W_{12}(q, \omega)$ proposed by Świerkowski et al. ${ }^{13,17}$ (SSG) as follows:

$$
\hat{W}_{S S G}(q, \omega)=\hat{v}_{\text {eff }}(q)+\hat{v}_{\text {eff }}(q) \hat{\chi}(q, \omega) \hat{v}_{\text {eff }}(q),
$$

where $v_{e f f}^{i j}(q)=v_{i j}(q)\left(1-G_{i j}(q)\right)$ are the effective Coulomb interactions and $G_{i j}(q)$ are intra- and inter-local-field corrections (LFCs) which take into account multiple scattering to infinite order between all components of the plasma compared with the RPA where these effects are neglected.

A more detailed analysis, which accounts for the vertex corrections associated with charge-charge fluctuation, was 
carried out for an electron gas in Refs. 14, 16, and 23, where Kukkonen-Overhauser-like effective interlayer interaction potential $^{15}$ was obtained by different approaches. In this scheme we have

$$
\hat{W}_{V S}(q, \omega)=\hat{v}_{e f f}(q)+\hat{v}_{e f f}(q) \hat{\chi}(q, \omega) \hat{v}_{e f f}(q)-\hat{U},
$$

with the elements of $\hat{U}$ defined by $v_{i j}(q) G_{i j}(q)$. The form of $W_{12}(q, \omega)$ within the Vignale and Singwi (VS) approach is similar to that in the self-consistent field approach of Singwi et al. ${ }^{12,13}$ (SSG) except for the last term. More clearly, the interlayer interaction in Eq. (5) is given by ${ }^{16,23}$

$$
\left.W_{12}(q, \omega)\right|_{V S}=\frac{v_{12}(q)\left(1-G_{12}(q)\right)}{\Delta(q, \omega)}-v_{12}(q) G_{12}(q),
$$

where

$$
\begin{aligned}
\Delta(q, \omega)= & {\left[1-v_{11}(q)\left(1-G_{11}(q)\right) \chi_{1}^{0}(q, \omega, T)\right] } \\
& \times\left[1-v_{22}(q)\left(1-G_{22}(q)\right) \chi_{2}^{0}(q, \omega, T)\right] \\
& -\left[v_{12}(q)\left(1-G_{12}(q)\right)\right]^{2} \chi_{1}^{0}(q, \omega, T) \chi_{2}^{0}(q, \omega, T) .
\end{aligned}
$$

Here $\chi_{k}^{0}(q, \omega, T)$ is noninteracting charge-charge response function at finite temperature. ${ }^{6}$

Another approximation scheme for screened bilayer 2D electron-electron interaction is proposed by Zheng and MacDonald $^{24}(\mathrm{ZM})$. In this scheme the screened electronelectron interaction potential is given as

$$
\hat{W}_{Z M}(q, \omega)=\left[1-\hat{\chi}^{0}(q, \omega, T) \hat{v}_{e f f}(q)\right]^{-1} \hat{v}(q) .
$$

This is derived essentially from a two-component generalization of the vertex function that enters in self-energy in the so-called $G W \Gamma$ approximation. However, because of the matrix nature of two-component systems there seems to be some ambiguity in such a construction. Note, for instance, that $\hat{W}_{Z M}$ is not a symmetric matrix for unmatched bilayer systems. Finally, we remark that VS, SSG, and ZM forms of the effective electron-electron interactions reduce to RPA if the LFCs are omitted.

As it is clear from Eqs. (4), (5), and (8) the local-field corrections are the fundamental quantities for an evaluation of the effective electron-electron interaction. Here, we intend to examine the interlayer potential of the Coulomb bilayer system by including correlation effects. To this purpose, we made use of the STLS approach both at zero (STLS0) and finite temperature (STLS) schemes. The STLS theory embodies correlations beyond the RPA approach and as an important improvement. In this approach the static LFC that accounts for correlation effects among carriers in the layers $k$ and $l$ is given by

$$
G_{k l}(q)=-\frac{1}{n} \int \frac{d \mathbf{q}^{\prime}}{(2 \pi)^{2}} \frac{\mathbf{q} \cdot \mathbf{k}}{q^{2}} \frac{v_{k l}\left(q^{\prime}\right)}{v_{k l}(q)}\left[S_{k l}\left(\left|\mathbf{q}-\mathbf{q}^{\prime}\right|\right)-\delta_{k l}\right],
$$

where $S_{k l}(q)$ is a static structure factor. The equations of motion for the Wigner distribution functions in a bilayer coupled with the linear-response theory yield in the approach of Singwi et al. ${ }^{12}$ the following expression for the densitydensity response functions:

$$
\chi_{k l}(q, \omega)=\frac{\chi_{k}^{0}(q, \omega, T)\left\{\delta_{k l}+(-1)^{\delta_{k l}} v_{k l}(q)\left(1-G_{k l}(q)\right) \chi_{l}^{0}(q, \omega, T)\right\}}{\Delta(q, \omega)} .
$$

The fluctuation-dissipation theorem leads to the static structure factor for a bilayer at finite temperature

$$
S_{k l}(q)=-\frac{\hbar}{\pi \sqrt{n_{k} n_{l}}} \int d \omega \operatorname{Im} \chi_{k l}(q, \omega) \operatorname{coth}\left(\frac{\hbar \omega}{2 k_{B} T}\right) .
$$

Equations (9)-(11) are solved numerically in a selfconsistent way for $G_{k l}(q)$ both at zero and finite temperature cases separately.

Another sophisticated method is based on Fermi hypernetted-chain approach (FHNC). Our strategy follows a similar approach to our recent works, ${ }^{25}$ which uses accurate intra- and interlayer static structure factors to build the localfield corrections. For this purpose we implement the selfconsistent Fermi hypernetted-chain approach ${ }^{26-28}$ at zero temperature in order to calculate the intra- and interlayer static structure factors incorporating the finite thickness effects in a quantum well. The latter effects are known to be important for the adequate description of the drag resistivity from a number of calculations. ${ }^{6,10,13,17}$ In what follows we explain the FHNC approximation and then outline our method to obtain the static local-field corrections, $G_{i j}(q)$, at zero temperature.

With the zero of energy taken at the chemical potential, the formally exact differential equation for the paircorrelation function, ${ }^{29} g_{\alpha \beta}(r)$, and following Chakraborty ${ }^{30}$ using the two-component plasma Jastrow-Slater variational theory involving FHNC approximation, reads

$$
\left[-\frac{\hbar^{2}}{m} \nabla^{2}+V_{\alpha \beta}^{e f f}(r)\right] \sqrt{g_{\alpha \beta}(r)}=0,
$$

where $m$ is electron mass and $V_{\alpha \beta}^{\text {eff }}(r)=v_{\alpha \beta}(r)+W_{\alpha \beta}^{B}(r)$ $+W_{\alpha \beta}^{F}(r)$. In Eq. (12) we decompose the effective potential into three terms $v_{\alpha \beta}(r), W_{\alpha \beta}^{B}$, and $W_{\alpha \beta}^{F}$ of which the last two terms take into account correlation and exchange effects, respectively; we substitute to the direct boson potential $W_{\alpha \beta}^{B}$ the one calculated by Chakraborty ${ }^{30}$ for a two-component 
Bose system using the static structure factors $S_{\alpha \beta}(k)$ of a Fermi system as follows:

$$
\begin{gathered}
W_{\alpha \alpha}^{B}(k)=-\frac{\hbar^{2} k^{2}}{4 m n_{\alpha}}\left[2 S_{\alpha \alpha}(k)-3+\left(S_{\bar{\alpha} \bar{\alpha}}^{2}(k)+S_{\alpha \bar{\alpha}}^{2}(k)\right) / \Gamma^{2}(k)\right] \\
W_{\alpha \bar{\alpha}}^{B}(k)=-\frac{\hbar^{2} k^{2}}{4 m \sqrt{n_{\alpha} n_{\bar{\alpha}}}}\left[2 S_{\alpha \bar{\alpha}}(k)-S_{\alpha \bar{\alpha}}(k)\left(S_{\bar{\alpha} \bar{\alpha}}(k)\right.\right. \\
\left.\left.+S_{\alpha \alpha}(k)\right) / \Gamma^{2}(k)\right] .
\end{gathered}
$$

Here $S_{\alpha \beta}(k)=\delta_{\alpha \beta}+\sqrt{n_{\alpha} n_{\beta}} \int\left[g_{\alpha \beta}(r)-1\right] \exp (i \mathbf{k} \cdot \mathbf{r}) d \mathbf{r}$ is the static structure factor and

$$
\Gamma(k)=S_{11}(k) S_{22}(k)-S_{12}^{2}(k) .
$$

Turning to the exchange term $W_{\alpha \beta}^{F}$, it is itself defined in order to make Eq. (12) exact and has a very complicated expression within the FHNC. ${ }^{26-28}$ However, in dealing with a onecomponent electron fluid, Kallio and Piilo ${ }^{31}$ have proposed a simple and efficient way to account for the antisymmetry of the fermion wave function. Their argument is immediately generalized to our two-component Fermi fluid, and leads to the requirement that, in the high density regime in both layers, the Hartree-Fock pair distribution functions are solution of Eq. (12). Following this prescription, it turns out that $W_{\alpha \beta}^{F}(k)$ is given by

$$
\begin{aligned}
W_{\alpha \alpha}^{F}(k)= & \int \frac{\hbar^{2}}{m} \frac{\nabla_{\mathbf{r}}^{2} \sqrt{g_{\alpha \alpha}^{\mathrm{HF}}(r)}}{\sqrt{g_{\alpha \alpha}^{\mathrm{HF}}(r)}} e^{i \mathbf{k r}} d \mathbf{r} \\
& +\frac{\hbar^{2} k^{2}}{4 m n_{\alpha}}\left\{2 S_{\alpha \alpha}^{\mathrm{HF}}(k)-3+\left[\frac{1}{S_{\alpha \alpha}^{\mathrm{HF}}(k)}\right]^{2}\right\},
\end{aligned}
$$

and $W_{\alpha \bar{\alpha}}^{F}(k)=0$. In Eq. (15) we used the fact that the Coulomb term in Eq. (12) becomes negligible in the HartreeFock limit with respect to the kinetic term.

Although the expression for the exchange potential in Eq. (15) is correct only for weakly coupled Fermi fluids, we shall assume in the following that it can yield useful results in our self-consistent calculations of the pair distribution functions with increasing coupling strength. ${ }^{29}$ As a broad qualitative argument in support of this assumption we may remark that the role of the statistics is expected to weaken with increasing coupling strength making the correlation term dominate on the exchange one. In Eq. (15) $S_{\alpha \alpha}^{\mathrm{HF}}(k)$ and $g_{\alpha \alpha}^{\mathrm{HF}}(r)$ are, respectively, the static structure factor and the intralayer pair distribution functions in the Hartree-Fock (HF) approximation namely,

$$
\begin{aligned}
S_{\alpha \alpha}^{\mathrm{HF}}(k)= & \frac{2}{\pi}\left[\arcsin \left(\frac{k}{2 k_{F_{\alpha}}}\right)+\frac{k}{2 k_{F_{\alpha}}} \sqrt{1-\left(\frac{k}{2 k_{F_{\alpha}}}\right)^{2}}\right] \\
& \times \vartheta\left(2 k_{F_{\alpha}}-k\right)+\vartheta\left(k-2 k_{F_{\alpha}}\right),
\end{aligned}
$$

and $g_{\alpha \alpha}^{\mathrm{HF}}(r)=1-2\left[j_{1}\left(r k_{F_{\alpha}}\right) / r k_{F_{\alpha}}\right]^{2}$ and $g_{\alpha \bar{\alpha}}^{\mathrm{HF}}(r)=1$, where $j_{1}$ is a spherical Bessel function, and $k_{F_{\alpha}}=\left(2 \pi n_{\alpha}\right)^{1 / 2}$.

It is evident that the insertion of Eqs. (13)-(16) into Eq. (12) allows a self-consistent calculation of the pair distribution functions and of the effective interactions. The fluctuation-dissipation theorem, which is of paramount im-
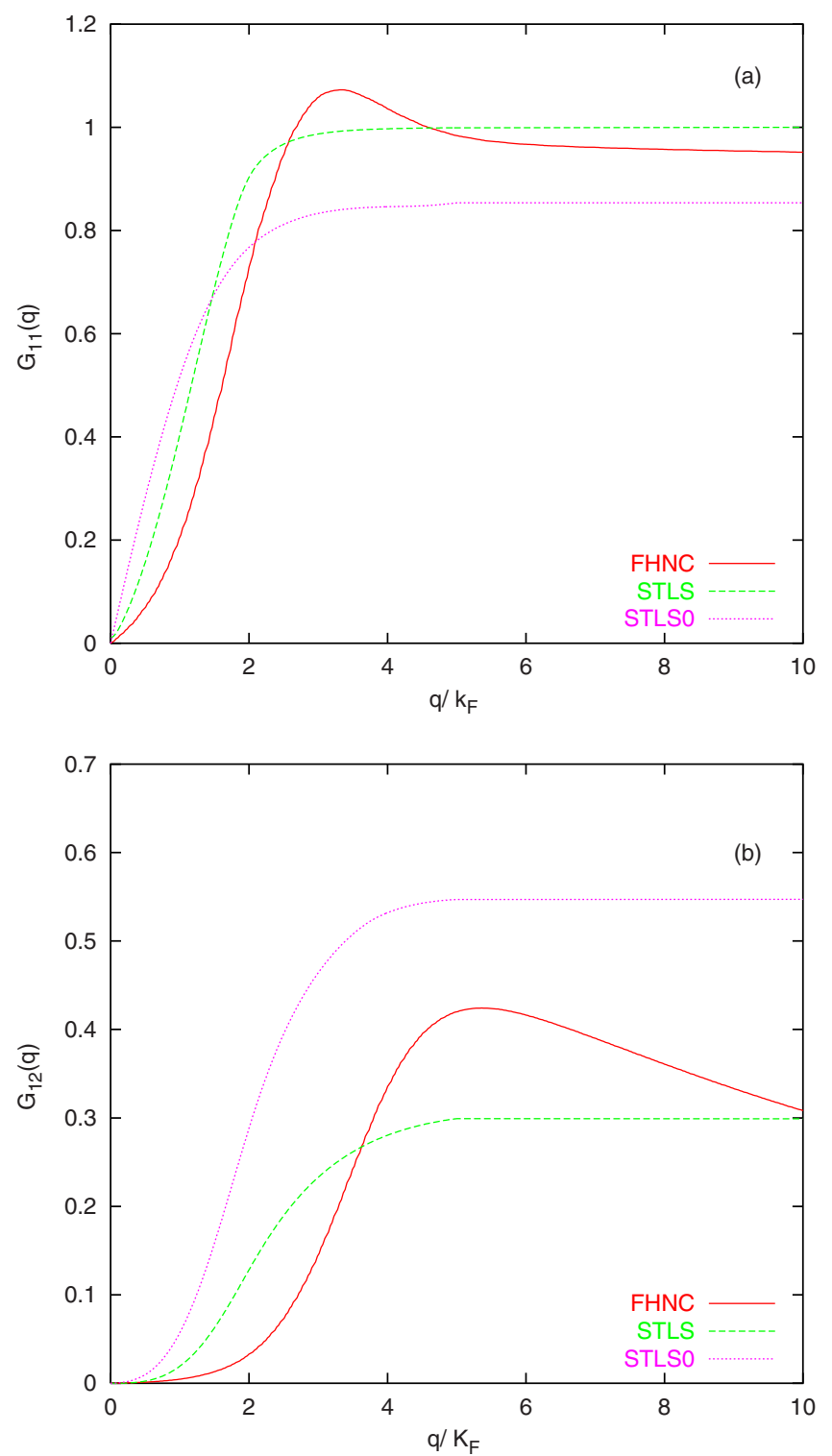

FIG. 1. (Color online) The local-field corrections (LFCs) in various models. (a) Intra layer LFC $G_{11}(q)$; (b) interlayer LFC $G_{12}(q)$. Solid, dashed, and dotted lines correspond to FHNC, STLS (at $T$ $=1 \mathrm{~K}$ ), and STLS0, respectively. The calculations are for equal density layers $\left(n=3.1 \times 10^{10} \mathrm{~cm}^{-2}\right)$ and sample parameters are as in Ref. 8.

portance for systems in equilibrium, relates the dynamic susceptibilities defined above to the static structure factors and allows one to determine the local-field corrections once the static structure factors are calculated by FHNC approach. ${ }^{25}$

\section{NUMERICAL RESULTS}

In this section we present our calculations for drag resistivity $\rho_{D}$ using the theoretical models described above and compare them with the recent experimental measurements. We proceed to illustrate our main numerical results, which are summarized in Figs. 1-7.

The effective interlayer interaction models which go beyond the RPA use local-field corrections as input. In Fig. 1, 

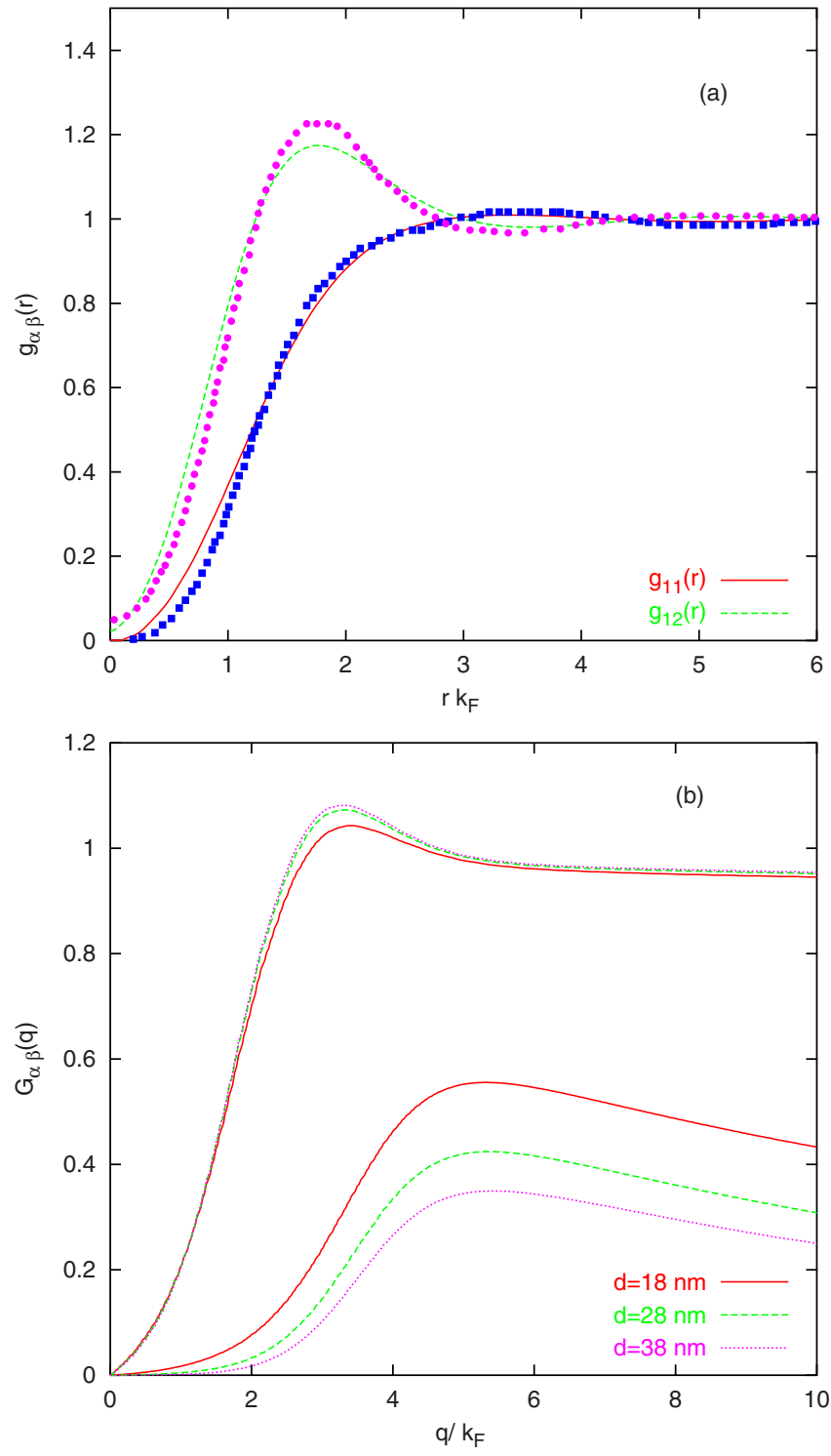

FIG. 2. (Color online) (a) The intra- and interlayer paircorrelation functions at $r_{s}=7.07$ calculated within the FHNC approach in comparison with QMC results of Ref. 17. (b) The intraand interlayer local-field corrections (LFCs) at $n=3.1$ $\times 10^{-10} \mathrm{~cm}^{-2}\left(r_{s}=3.25\right)$ calculated within the FHNC approach for different interlayer distances $d$.

we display the typical behavior of intra- and interlayer LFCs $G_{11}(q)$ and $G_{12}(q)$, respectively. We note that whereas the LFCs in the STLS approach have a monotone $q$ dependence, FHNC approach yields a peaked structure. Such a structure in static LFCs is well known from quantum Monte Carlo simulations $^{32}$ and it is thought to represent the correlation effects correctly. Thus, differences in LFCs will evidently play an important role in the drag resistivity. We also remark that there is a considerable difference between zero and finite temperature (at $T=1 \mathrm{~K}$ ) STLS calculations especially for the interlayer LFC, $G_{12}(q)$. We believe that within our calculational scheme the FHNC approach yields the most accurate LFCs. To illustrate our point, we compare the intra- and interlayer pair-correlation functions $g_{\alpha \beta}(r)$ resulting from FHNC calculations and quantum Monte Carlo (QMC) simulations ${ }^{17}$ in Fig. 2(a). We note that the agreement is very good. The STLS scheme does not reproduce well the peak structure in $g_{11}(r)$ at this density which corresponds to $r_{s}$ $=7.07$. We have also looked at the interlayer distance $d$ dependence of the LFCs within the FHNC approach. Figure 2(b) shows intra- and interlayer LFCs for various values of $d$ at a layer density $n=3.1 \times 10^{-10} \mathrm{~cm}^{-2}$. We have also used the finite quantum-well widths corresponding to the experimental sample of Kellogg et al. ${ }^{8}$ As expected, the intralayer LFC $G_{11}(q)$ is not affected much as $d$ changes, whereas the interlayer LFC $G_{12}(q)$ becomes smaller in magnitude as $d$ increases, reflecting the weakened Coulomb correlations. Similar qualitative results have also been found in a bilayer STLS calculation. ${ }^{33}$

In Figs. 3 and 4 we show the calculated drag resistivity as a function of temperature for various theoretical models of effective interlayer interaction (i.e., models denoted as VS, SSG, and ZM) with different LFCs (i.e., schemes denoted as FHNC, STLS, and STLS0) at layer densities $3.1 \times 10^{10}$ and $3.8 \times 10^{10} \mathrm{~cm}^{-2}$ and compare them with the experimental results of Kellogg et al. ${ }^{8}$ The experimental data were obtained for bilayer GaAs-AlGaAs heterostructures for two identical infinite layers of electrons separated by $d=280 \AA$ and with a double quantum well of widths $L=180 \AA$. In all our results, the drag resistivity calculated within the VS interlayer potential is larger than the one calculated within the SSG approximation. It means that the value of $U$ increases with increasing $G_{12}(q)$, and VS potential in Eq. (5) becomes highly different from the SSG potential given by Eq. (4). The static LFCs which are constructed within the FHNC approach together with the electron-electron interlayer potential calculated within VS and SSG approaches give results in quite good agreement with experimental measurements especially in the low temperature regime below the plasmon-mediated drag. In these figures, the RPA results underestimate the experimental results. Therefore, after the inclusion of manybody effects correctly (such as using FHNC), the drag resistivity is in good quantitative agreement with experimental results. The LFCs in STLS scheme yield an overestimate of drag resistivity when it is calculated using the VS and SSG interlayer potentials, $W_{12}(q, \omega)$. From the physical point of view, correlation effects suppress the energies of both the acoustic and optical plasmons, while finite temperature effects tend to increase the energies. From this cancellation, the STLS0/SSG gives results close to the experimental data in comparison to STLS/SSG. Furthermore, the value of intralayer LFC at finite temperature at a given $q<2 k_{F}$ value is smaller than the intralayer LFC at zero temperature in STLS0 scheme and this yields to have larger plasmon contribution in drag resistivity when one employs the zero temperature LFCs. Furthermore, the interlayer LFC at zero temperature is larger than the one at finite temperature, thus the drag resistivity in STLS0/VS is further overestimated than in STLS/VS approach.

Figure 5 shows the log-log plot of the drag resistivity $\rho_{D}$ as a function of layer density at $T=1$ and $4 \mathrm{~K}$. For comparison with recent calculations of drag resistivity by Badalyan et al. ${ }^{17}$ we extract their results from their Fig. 15 (denoted in 

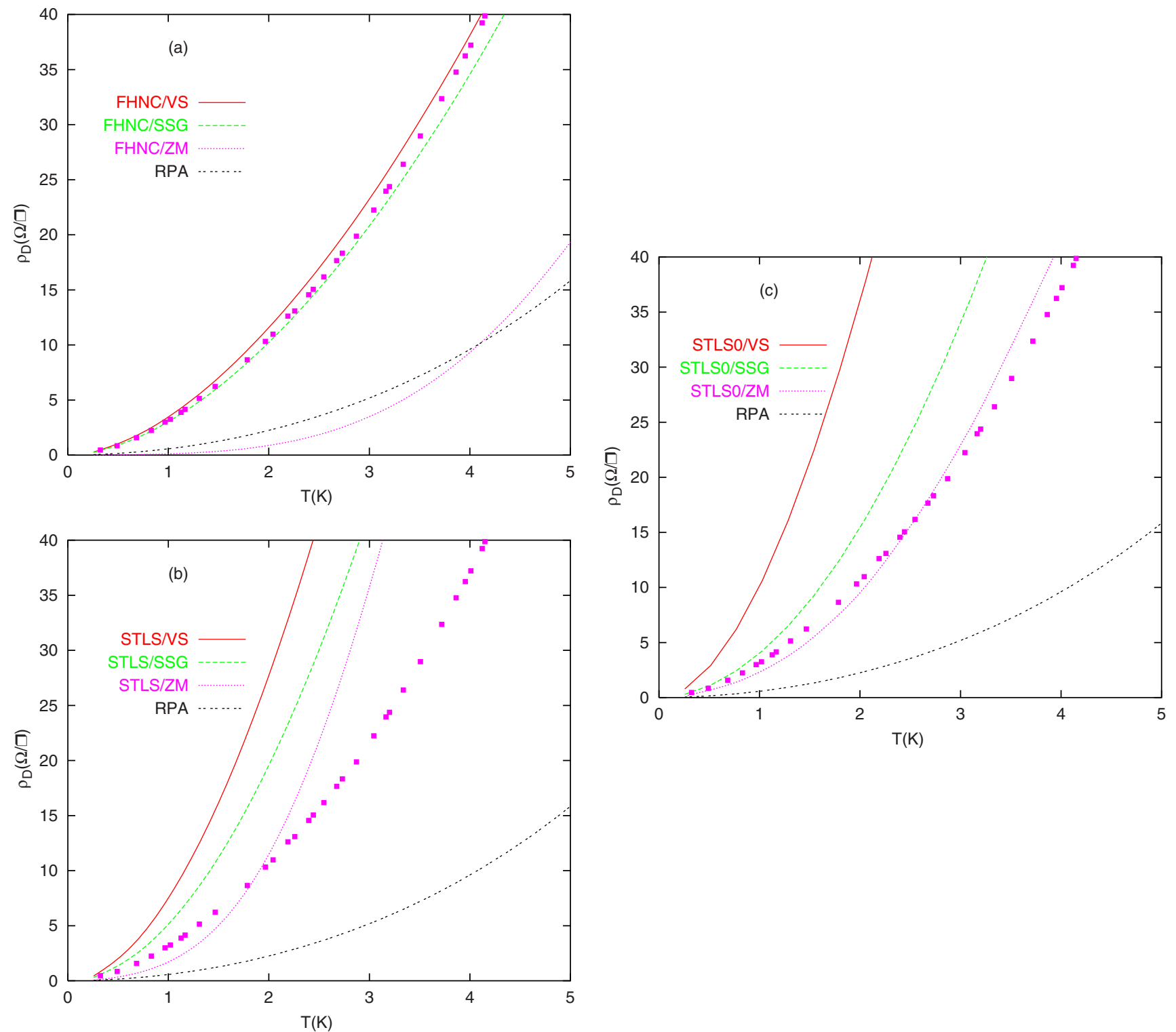

FIG. 3. (Color online) The temperature dependence of the drag resistivity for the identical bilayer electron-electron systems for $n=3.1$ $\times 10^{10} \mathrm{~cm}^{-2}\left(r_{s}=3.25\right)$. The full boxes are the experimental data of Ref. 8. (a) FHNC, (b) STLS, and (c) STLS0 local-field corrections are used in conjunction with different screened interlayer interaction models.

our Fig. 5 by BKVS) and compare them with the results of our calculations, mainly FHNC/VS and FHNC/SSG. Evidently, our FHNC calculation produces better agreement with experiment in the whole range of density compared to all other approximations.

The low temperature behavior of drag resistivity $\rho_{D}$ for the samples of Kellogg et al. ${ }^{8}$ is important in understanding the interaction effects. The low density and close interlayer spacing such that $k_{F} d \lesssim 1$ implies significant contributions of backward scattering processes to $\rho_{D}$ and predicts deviations from the usual $T^{2}$ dependence. ${ }^{2}$ These deviations expected to be in the form $\sim T^{2} \ln T$ are difficult to be assessed, but the sensitivity of $\rho_{D}$ to layer densities has been noted. In Fig. 6 we show the scaled drag resistivity $\rho_{D} / T^{2}$ as a function of temperature for $n=3.8 \times 10^{10} \mathrm{~cm}^{-2}$ and $n=2.3 \times 10^{10} \mathrm{~cm}^{-2}$. The drag resistivity including the FHNC local-field corrections through the various screened interlayer interaction models is compared with RPA. We note that VS and SSG screened interlayer interaction models reproduce the upturn behavior of $\rho_{D} / T^{2}$ at low temperature observed in the experiments by Kellogg et ll $^{8}$ On the other hand, ZM model predicts an opposite behavior in contradiction with experiments. The increase in $\rho_{D} / T^{2}$ at low temperatures due to exchangecorrelation effects was first analyzed by Badalyan et al. ${ }^{17}$ where they used the static local-field corrections in this temperature regime. Similar enhancement in scaled drag resistivity was also obtained by Hwang et al. ${ }^{34}$ in their calculation on bilayer hole systems in connection with the experiments of Pillarisetty et al. ${ }^{9}$ Our comparative study thus provides information as to which form of screened interlayer interaction is more suitable in describing drag experiments at low density.

Finally, we display the interlayer distance dependence of the many-body correlation effects on drag resistivity in Fig. 

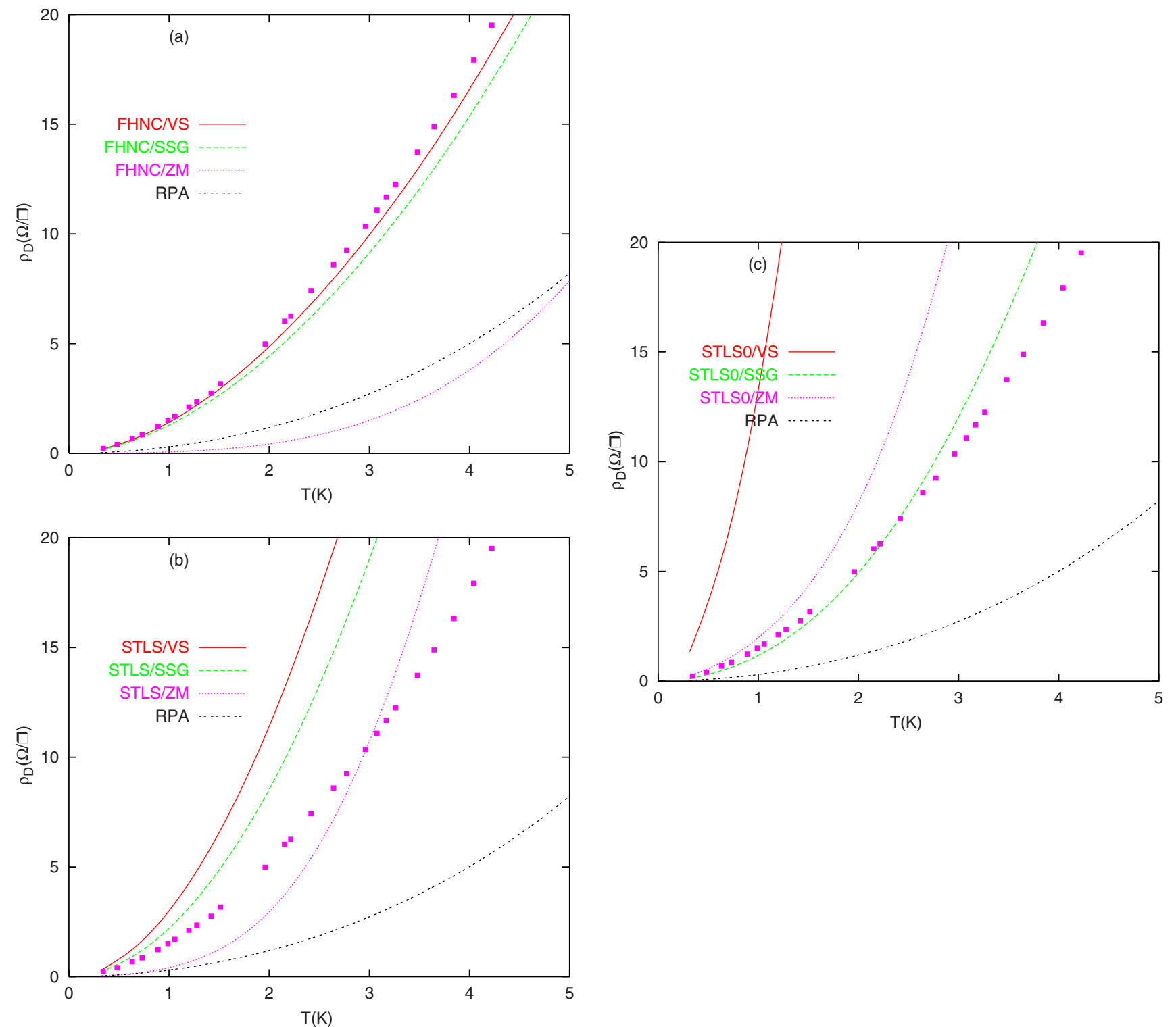

FIG. 4. (Color online) The temperature dependence of the drag resistivity for the identical bilayer electron-electron systems for $n=3.8 \times 10^{10} \mathrm{~cm}^{-2}\left(r_{s}=2.93\right)$. The full boxes are the experimental data of Ref. 8. (a) FHNC, (b) STLS, and (c) STLS0 local-field corrections are used in conjunction with different screened interlayer interaction models.

7. When the layer separation is decreased, interlayer Coulomb interaction enhances and this leads to an increase in drag resistivity. Because the experiment of Kellogg et al. ${ }^{8}$ did not measure $\rho_{D}$ for samples with different $d$ values, we are not able to make a systematic comparison.

In the examples above we have seen that the local-field factors play a significant role in the quantitative description of the measured drag resistivity. It is important to remark that the drag resistivity is calculated for electron-electron interaction only and we ignore other scattering processes (i.e., impurities, phonons, etc.) which may be effective in realistic situations. In general, the theoretical prediction by the results of Eq. (2) should yield values below the experimental measurements for drag resistivity in which all scattering processes are included. ${ }^{11,22,35}$ Since our calculations already provide a very good agreement with Kellogg et al. ${ }^{8}$ we can argue that phonon and impurity effects are not very impor- tant for these samples. The phonon contribution is identified by the peak in $\rho_{D} / T^{2}$ which occurs when the average thermal phonon wave vector becomes comparable to $2 k_{F}$. The data of Kellogg et al. ${ }^{8}$ do not show such a peak. Disorder in general enhances the drag resistivity and, in particular, when the electron or hole layers are close to metal-insulator transition it plays a very important role. ${ }^{9}$ We have not systematically studied the disorder effect here but judging from the results of our comparison to the data of Kellogg et al. ${ }^{8}$ we surmise that it is not significant.

We also note that we model the finite width of experimental sample by an infinite square well which modifies the bare potentials by a form factor. The effect of correct form factor and its parameters (barrier height, etc.) obtained by well geometry may be crucial in the final results for drag resistivity. We have not done a self-consistent calculation of a realistic bilayer structure to test this. Variations up to $20 \%$ in the 

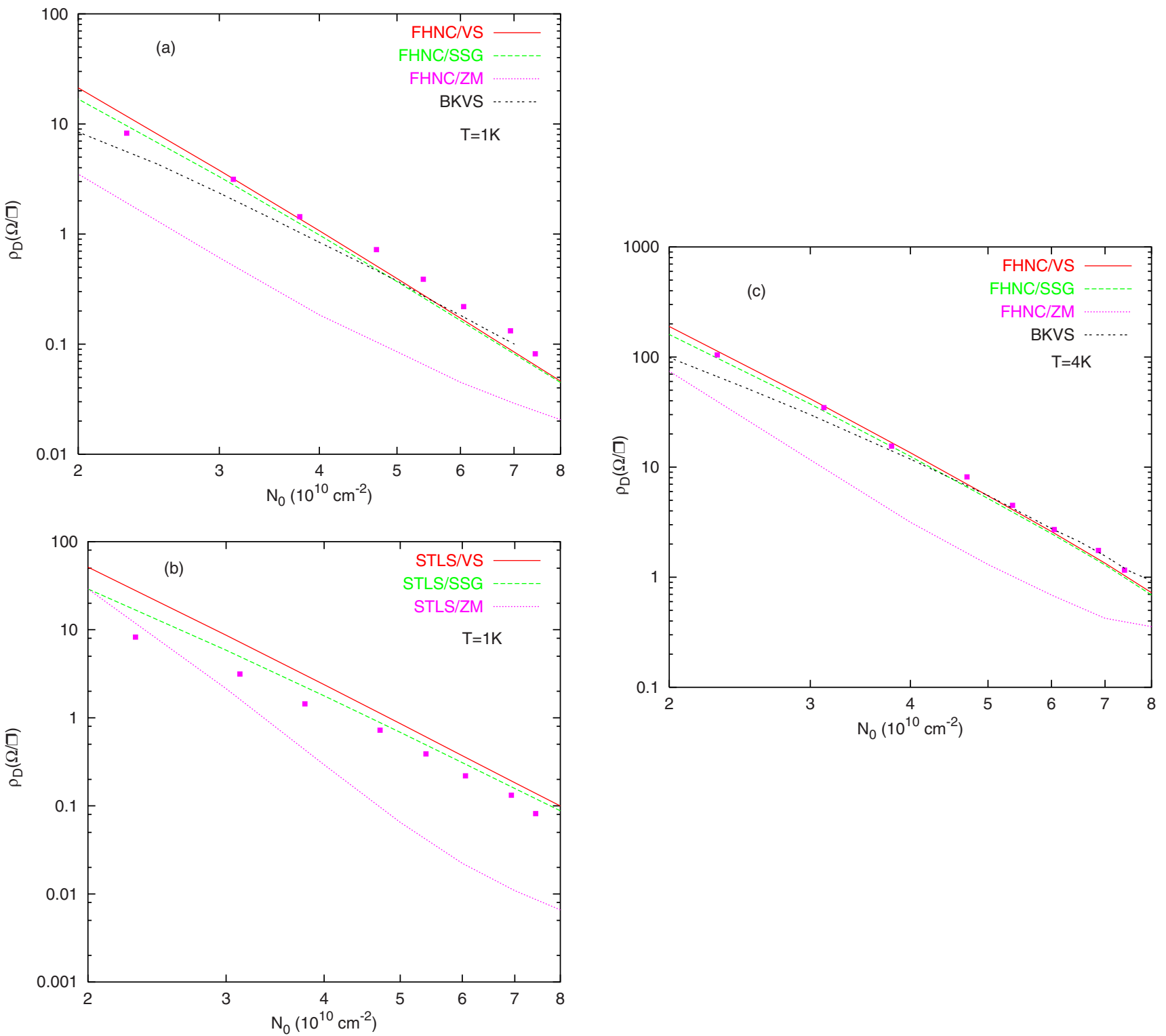

FIG. 5. (Color online) The density dependence of the drag resistivity for the identical bilayer electron-electron systems at $T=1$ and $4 \mathrm{~K}$ in a log-log plot. The full boxes are the experimental data of Ref. 8. BKVS refers to Ref. 17. (a) FHNC and (b) STLS local-field corrections are used in conjunction with different screened interlayer interaction models at $T=1 \mathrm{~K}$. (c) Same as (a) at $T=4 \mathrm{~K}$.

quantum-well width $L$, however, does not seem to affect the drag resistivity at low temperatures.

\section{SUMMARY}

We have investigated the performance of various models of interlayer electron-electron interactions on the temperature dependence of drag resistivity. Such models going beyond the RPA are necessary to account for increasing correlation effects at low density. A major input to construct an effective interlayer interaction is local-field corrections. We have considered the self-consistent field approach of Singwi et al. ${ }^{12}$ both at zero and finite temperatures and FHNC formalism to obtain intra- and interlayer local-field corrections. Our calculations compared with relevant experimental results of Kellogg et al. ${ }^{8}$ demonstrate the importance of including cor- relation effects correctly in the drag resistivity formula. The effective interaction model developed by Vignale and Singwi ${ }^{16}$ supplemented by local-field corrections from FHNC approach provides very good quantitative agreement with experiments. Furthermore, previous application ${ }^{14}$ of the VS effective interaction model with simplified local-field corrections finds justification in the present calculations. In the temperature range of the experiments of Kellogg et al. ${ }^{8}$ where the plasmon contribution should not be significant, static local-field corrections account for the observed drag resistivity.

It would be of interest to develop frequency dependent local-field corrections at a similar level of sophistication presented in this work to investigate the dynamic correlations. They are expected to be important for the plasmon-mediated drag occurring at high temperatures $\left(T \sim T_{F}\right)$ as discussed by 

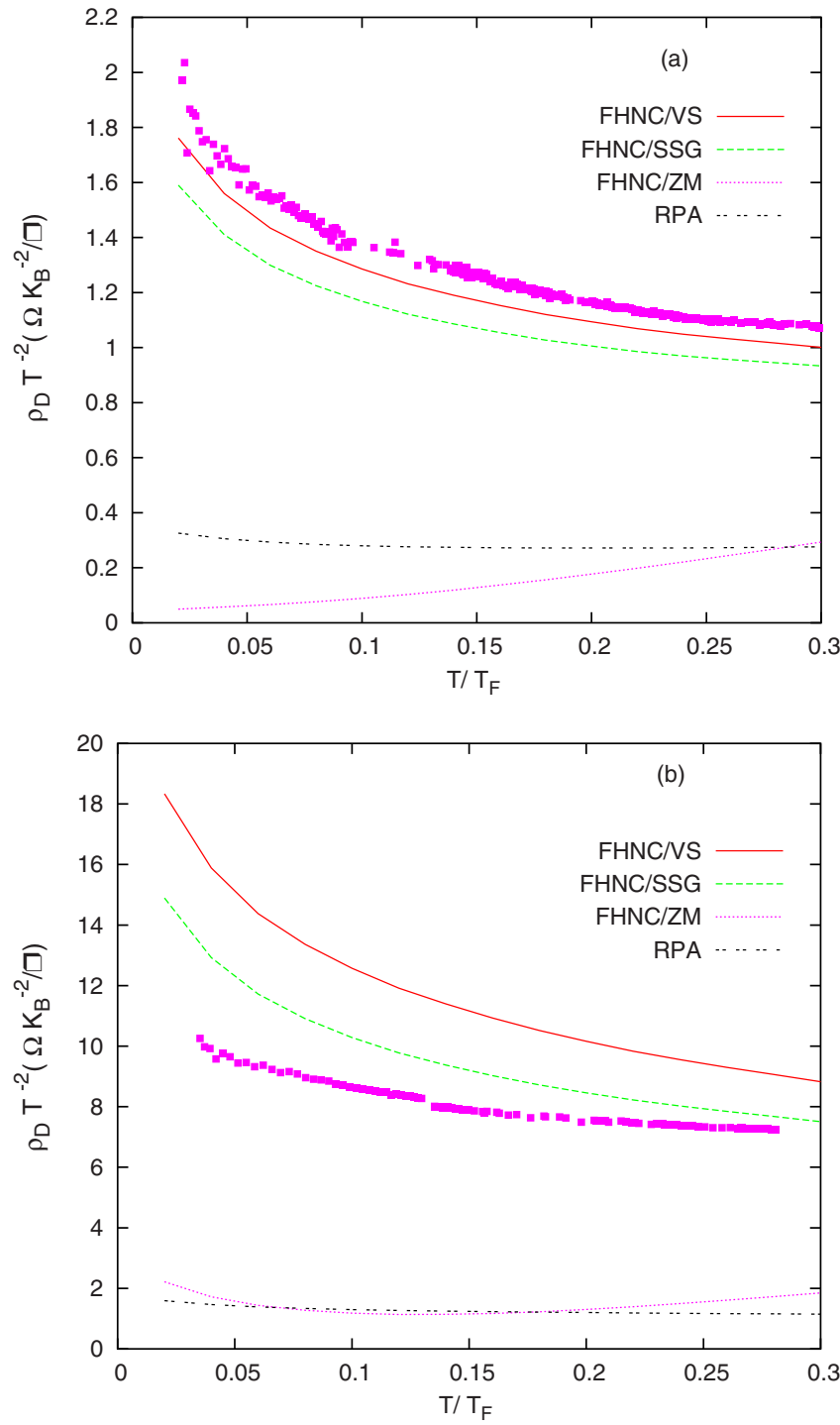

FIG. 6. (Color online) The scaled drag rate $\rho_{D} / T^{2}$ as a function of temperature for (a) $n=3.8 \times 10^{10} \mathrm{~cm}^{-2}\left(r_{s}=2.93\right)$ and (b) $n=2.3$ $\times 10^{10} \mathrm{~cm}^{-2}\left(r_{s}=3.77\right)$. The full boxes are the experimental data of Ref. 8. FHNC local-field corrections used in conjunction with different interlayer interaction models are compared with RPA.

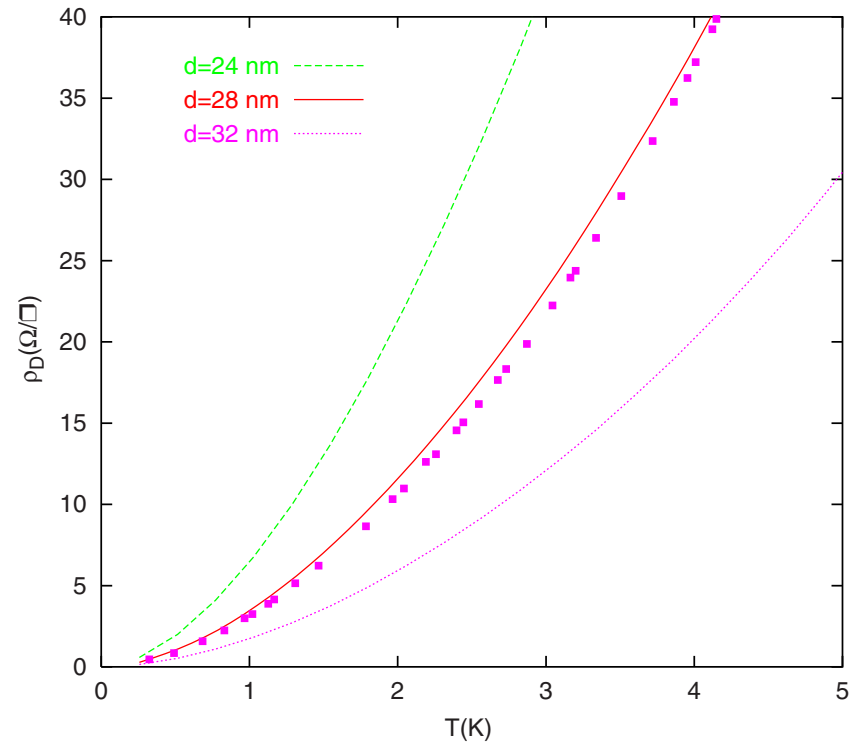

FIG. 7. (Color online) The temperature dependence of the drag resistivity for a bilayer electron system with layer density $n=3.1$ $\times 10^{10} \mathrm{~cm}^{-2}\left(r_{s}=3.25\right)$. The full boxes are the experimental data of Ref. 8 at the same density and $d=280 \AA$.

Flensberg and $\mathrm{Hu}^{6}$ and most recently by Badalyan et al. ${ }^{17}$ Especially, single- and multipair decay mechanisms when properly incorporated in the dynamic correlations may explain the observed behavior ${ }^{7}$ of drag resistivity in bilayers with unmatched densities. Another possible direction is to study the phonon-mediated drag for low density systems which should be effective at layer separations larger than those considered in this work.

\section{ACKNOWLEDGMENTS}

We are grateful to G. Vignale for illuminating discussions and comments. We thank B. Y.-K. Hu for discussions at an earlier stage. This work is supported by TUBITAK (106T052) and TUBA.
${ }^{1}$ For a recent review of the current status of the field, see S. V. Kravchenko and M. P. Sarachik, Rep. Prog. Phys. 67, 1 (2004).

${ }^{2}$ T. J. Gramila, J. P. Eisenstein, A. H. MacDonald, L. N. Pfeiffer, and K. W. West, Phys. Rev. Lett. 66, 1216 (1991); Phys. Rev. B 47, 12957 (1993); U. Sivan, P. M. Solomon, and H. Shtrikman, Phys. Rev. Lett. 68, 1196 (1992); H. Rubel, E. H. Linfield, D. A. Ritchie, K. M. Brown, M. Pepper, and G. A. C. Jones, Semicond. Sci. Technol. 10, 1229 (1996).

${ }^{3}$ For a review, see A. G. Rojo, J. Phys.: Condens. Matter 11, R31 (1999).

${ }^{4}$ A. Punnoose and A. M. Finkelstein, Science 310, 289 (2005).

${ }^{5}$ N. P. R. Hill, J. T. Nicholls, E. H. Linfield, M. Pepper, D. A. Ritchie, G. A. C. Jones, Ben Yu-Kuang Hu, and K. Flensberg,
Phys. Rev. Lett. 78, 2204 (1997).

${ }^{6}$ K. Flensberg and Ben Yu-Kuang Hu, Phys. Rev. B 52, 14796 (1995).

${ }^{7}$ H. Noh, S. Zelakiewicz, X. G. Feng, T. J. Gramila, L. N. Pfeiffer, and K. W. West, Phys. Rev. B 58, 12621 (1998).

${ }^{8}$ M. Kellogg, J. P. Eisenstein, L. N. Pfeiffer, and K. W. West, Solid State Commun. 123, 515 (2002).

${ }^{9}$ R. Pillarisetty, H. Noh, D. C. Tsui, E. P. De Poortere, E. Tutuc, and M. Shayegan, Phys. Rev. Lett. 89, 016805 (2002).

${ }^{10}$ A. P. Jauho and H. Smith, Phys. Rev. B 47, 4420 (1993).

${ }^{11}$ L. Zheng and A. H. MacDonald, Phys. Rev. B 48, 8203 (1993).

${ }^{12}$ K. S. Singwi, M. P. Tosi, R. H. Land, and A. Sjölander, Phys. Rev. 176, 589 (1968) 
${ }^{13}$ L. Świerkowski, J. Szymanśki, and Z. W. Gortel, Phys. Rev. Lett. 74, 3245 (1995); Phys. Rev. B 55, 2280 (1997).

${ }^{14}$ A. Yurtsever, V. Moldoveanu, and B. Tanatar, Solid State Commun. 125, 575 (2003).

${ }^{15}$ C. A. Kukkonen and A. W. Overhauser, Phys. Rev. B 20, 550 (1979).

${ }^{16}$ G. Vignale and K. S. Singwi, Phys. Rev. B 31, 2729 (1985).

${ }^{17}$ S. M. Badalyan, C. S. Kim, G. Vignale, and G. Senatore, Phys. Rev. B 75, 125321 (2007).

${ }^{18}$ S. De Palo, F. Rapisarda, and G. Senatore, Phys. Rev. Lett. 88, 206401 (2002) and references therein.

${ }^{19}$ G. Vignale and A. H. MacDonald, Phys. Rev. Lett. 76, 2786 (1996); Ben Yu-Kuang Hu, ibid. 85, 820 (2000).

${ }^{20}$ D. S. Kainth, D. Richards, H. P. Hughes, M. Y. Simmons, and D. A. Ritchie, J. Phys.: Condens. Matter 12, 439 (2000).

${ }^{21}$ A. Kamenev and Y. Oreg, Phys. Rev. B 52, 7516 (1995).

${ }^{22}$ K. Flensberg, Ben Yu-Kuang Hu, A.-P. Jauho, and J. M. Kinaret, Phys. Rev. B 52, 14761 (1995).

${ }^{23}$ C. F. Richardson and N. W. Aschroft, Phys. Rev. B 55, 15130 (1997).

${ }^{24}$ L. Zheng and A. H. MacDonald, Phys. Rev. B 49, 5522 (1994).

${ }^{25}$ R. Asgari, A. L. Subasi, A. A. Sabouri-Dodaran, and B. Tanatar, Phys. Rev. B 74, 155319 (2006); R. Asgari and B. Tanatar, ibid. 74, 075301 (2006); R. Asgari, A. Esmilian, and B. Tanatar, Solid State Commun. 141, 595 (2007); R. Asgari, ibid. 141, 563
(2007).

${ }^{26}$ L. J. Lantto and P. J. Siemens, Nucl. Phys. A 317, 55 (1979); L. J. Lantto, Phys. Rev. B 36, 5160 (1987).

${ }^{27}$ J. G. Zabolitzky, Phys. Rev. B 22, 2353 (1980).

${ }^{28}$ E. Krotscheck and M. Saarela, Phys. Rep. 232, 1 (1993).

${ }^{29}$ S. Abedinpour, R. Asgari, M. Polini, and M. P. Tosi, Solid State Commun. 144, 65 (2007); B. Davoudi, R. Asgari, M. Polini, and M. P. Tosi, Phys. Rev. B 68, 155112 (2003).

${ }^{30}$ T. Chakraborty, Phys. Rev. B 25, 3177 (1982); 26, 6131 (1982); T. Chakraborty, A. Kallio, L. J. Lantto, and P. Pietiläinen, ibid. 27, 3061 (1983).

${ }^{31}$ A. Kallio and J. Piilo, Phys. Rev. Lett. 77, 4237 (1996).

${ }^{32}$ G. Senatore, S. Moroni, and D. M. Ceperley, in Quantum Monte Carlo Methods in Physics and Chemistry, edited by M. P. Nightingale and C. J. Umrigar (Kluwer, Dordrecht, 1999), p. 183; S. Moroni, D. M. Ceperley, and G. Senatore, Phys. Rev. Lett. 69, 1837 (1992).

${ }^{33}$ L. Liu, L. Swierkowski, D. Neilson, and J. Szymanski, Phys. Rev. B 53, 7923 (1996).

${ }^{34}$ E. H. Hwang, S. Das Sarma, V. Braude, and A. Stern, Phys. Rev. Lett. 90, 086801 (2003).

${ }^{35}$ Ben Yu-Kuang Hu, Phys. Rev. B 57, 12345 (1998); K. Guven and B. Tanatar, ibid. 56, 7535 (1997); B. Tanatar, ibid. 58, 1154 (1998). 